\title{
Common genetic polymorphisms of adenosine A2A receptor do not influence response to regadenoson
}

Mark Berlacher, Ronald Mastouri, Santosh Philips, Todd C Skaar \& Rolf P Kreutz

\begin{abstract}
Introduction:

Hemodynamic response to regadenoson varies greatly, and underlying mechanisms for variability are poorly understood. We hypothesized that 5 common variants of A2A receptor are associated with altered response to regadenoson.

Methods:

Consecutive subjects ( $n=357$ ) undergoing resting regadenoson nuclear stress imaging were enrolled. Genotyping was performed using Taqman based assays for rs5751862, rs229838, rs3761422, 2267076 and rs5751876.
\end{abstract}

\section{Results:}

There was no significant difference in heart rate or blood pressure between different genotypes following regadenoson administration. There was also no significant difference in myocardial ischemia detected by nuclear perfusion imaging as defined by summed difference score, or in self-reported side effects among the genotypes tested.

\section{Conclusions:}

The common A2A variants studied are not associated with variability in hemodynamic response to regadenoson or variability in detection of ischemia with nuclear perfusion stress imaging. 


\section{Introduction:}

Since its FDA approval in 2008, regadenoson has largely replaced adenosine as the principle agent used in pharmacological cardiac stress testing. Regadenoson is an adenosine analogue and selective A2A adenosine receptor agonist which causes vasodilation of arteriolar vascular smooth muscles cells and myocardial hyperemia, leading to the desired cardiac state for myocardial perfusion imaging (MPI). Compared to its predecessor, regadenoson produces hyperemia with more rapid onset (30 seconds) and for a longer time period (two to five minutes) [1]. In addition, the ADVANCE-MPI 1 and 2 randomized trials demonstrated noninferiority for regadenoson to adenosine when comparing efficacy for detection of reversible perfusion defects. Regadenoson was also associated with a decreased overall symptom score (which included dyspnea, chest pain and flushing), attributed to the selective activation of adenosine receptor A2A without acting on adenosine receptors $\mathrm{A} 1, \mathrm{~A} 2 \mathrm{~B}$ or $\mathrm{A} 3$ [2].

Despite the selective activity of regadenoson, there remains considerable interpatient variability in hemodynamic response and reported side effects associated with its use[3, 4]. We recently reported the association of adenosine monophosphate deaminase1(AMPD-1) polymorphism with increased incidence of reported adverse effects during regadenoson administration.

Several genetic variants of A2A receptor have been previously described. Single nucleotide polymorphisms (SNP) of A2A have been associated with functional differences in psychiatric and neurological phenotypes [5]. Several A2A variants (rs 5751862, rs2298383, rs3761422) were found to be associated with anxiety related personality scores. In particular rs5751876 has been associated with Gilles de la Tourette syndrome, caffeine 
induced anxiety, panic disorder with agoraphobia, and adverse effects in nonacute schizophrenia[6-9]. Levodopa-induced dyskinesis in Parkinson patients was also found to be associated with diplotypes of two A2A variants (rs2298383 and rs3761422)[10]. The pharmacodynamic impact of these common receptor variants with suggested functional phenotype variability on regadenoson response has not been previously examined.

We hypothesized that common $\mathrm{A} 2 \mathrm{~A}$ receptor polymorphisms are associated with altered hemodynamic response to regadenoson.

\section{Methods}

Patients

The study protocol was approved by the institutional review board (IRB) and a written consent was obtained for all patients enrolled.

All subjects who were scheduled for regadenoson nuclear stress testing were eligible for the study. Those who underwent a combination of exercise and nuclear pharmacologic stress testing with regadenoson were excluded from our analysis. Information on medical history, risk factors, and demographics were collected by interview and chart review.

\section{Genotyping}

The Qiagen Flexigene DNA Kit \#51206 was used for genomic DNA extraction following the protocol for isolation of DNA from 100-500 $\mu$ l buffy coat (Germantown, USA). SNPs associated with ADORA2A including rs5751862, rs229838, rs3761422, 2267076 and rs5751876 were analyzed using an open array genotyping platform (Life Technologies, 
Grand Island, NY, USA) according to the manufacturer's instructions. Amplification of alleles of interest were amplified using sequence-specific primers along with allele-specific TaqMan ${ }^{\circledR}$ probes (Applied Biosystems, Foster City, CA). Individual genotypes were determined using allelic discrimination.

\section{Regadenoson administration}

A dose of $0.4 \mathrm{mg}$ of regadenoson was administered through peripheral venous access over a period of ten seconds, followed by a saline flush of five mL over another ten seconds. Study Measurements

Systolic blood pressure (SBP), and heart rate (HR) were recorded prior to regadenoson infusion, then at one minute, and three minutes following infusion. Selfreported adverse symptoms were recorded. Primary outcomes were change in HR, and change in SBP. Change in HR was defined as the difference between the peak postadministration HR and the baseline HR. Change in SBP was defined as difference of baseline SBP minus $1 \mathrm{~min}$ or $3 \mathrm{~min}$ SBP following regadenoson administration.

Secondary outcomes included incidence of symptoms including abdominal pain, headache, nausea, dyspnea, flushing, dizziness and chest pain.

Nuclear myocardial perfusion imaging was analyzed using a standard 17 segment model. Summed stress score (SSS), summed rest score (SRS), and summed difference scores (SDS) were recorded[11].

Statistical Analysis

SPSS software, version 23.0 (IBM, Chicago, IL) was used to perform statistical analyses. A p-value of less than 0.05 was used to define statistical significance. Values reported represent the mean \pm standard deviation, unless specified otherwise. All statistical 
tests were two-sided. Continuous variables were compared using unpaired two-sided Student's t-tests, and analysis of variance testing (ANOVA) for multiple group testing. Categorical variables were compared using chi-square test. Haploview software was used for haplotype analysis (www.broadinstitute.org/haploview). Linkage disequilibrium (LD) between variants in same gene was expressed as $\mathrm{D}^{\prime}$ coefficients.

\section{Results}

A total of 357 patients underwent regadenoson stress testing and successful genotyping and were included in the study. Genotype distribution was consistent with Hardy-Weinberg equilibrium ( $p>0.05$ ) (Table 1). There was close linkage disequilibrium between rs229838, rs3761422, 2267076 and rs5751876 (Figure 1.)

The mean age was 58 years with 55\% being female and majority (71\%) Caucasian. Table 2 summarizes the baseline characteristics of the study population.

Genotype distribution for rs5751862 and rs2298383 was significantly different between Caucasian and African-American subjects, with lower prevalence of the minor allele variant among African Americans (Table 2). In addition, carriers of the minor allele variant for rs2298383, rs3761422, rs2267076, and rs5751876 had a higher prevalence of congestive heart failure. Chronic obstructive pulmonary disease was more common in minor allele carriers for rs2267076.

There was no significant difference in incidence of self-reported side effects between noncarriers and carriers of the minor allele variants studied (rs5751862, rs229838, rs3761422, 2267076 and rs5751876) (Table 3.). Symptoms reported included abdominal pain, headache, nausea, dyspnea, flushing, dizziness and chest pain. 
Baseline BP and heart rate were not significantly different between genotypes for all variants studied (Table 4.). After regadenoson administration the average rise in heart rate was 29.7 beats/min. Mean change in SBP at $1 \mathrm{~min}$ was $-2.7 \pm 18 \mathrm{mmHg}$ and

$-2.5 \pm 19 \mathrm{mmHg}$ at $3 \mathrm{~min}$. There was no significant difference in change of heart rate or SBP after regadenoson administration between genotypes of variants studied (Table 4).

The summed stress score (SSS) was higher in carriers of the minor allele variant of rs2267076, but not significantly different between different genotypes of rs5751862, rs229838, rs3761422, and rs5751876. The summed resting score (SRS) was also higher in carriers of the minor allele of rs2267076 and rs3761422. However, the summed difference score was not significantly different among any of the genotypes for each of the variant studied.

Since it has been reported that diabetes mellitus enhances A2A receptor response, we have included a subgroup analysis with only non-diabetic subjects[12]. This did not demonstrate any significant difference in baseline and post stress hemodynamic measurements, nor nuclear perfusion scores (supplemental data).

\section{Discussion}

Adenosine is an endogenous purine nucleoside that is known to affect cardiac myocyte activity via four structurally related G-protein coupled receptors (ADORA1, ADORA2A, ADORA2B and ADORA3). Levels of adenosine in the blood have been shown to affect heart rate, cardiac conduction impulse, coronary perfusion, remodeling of cardiovascular tissue and cardiac vascular resistance to adverse insult [13]. Thus, the activity of these receptors plays an important role in overall cardiovascular function. 
Regadenoson, a selective ADORA2A agonist, has largely replaced adenosine as the primary agent for pharmacologic cardiac stress testing. Certain receptor polymorphisms of ADORA2A have already been linked with various noncardiac conditions [5-9] including caffeine induced anxiety [14], childhood encephalopathy [15] and proliferative diabetic retinopathy [16]. Similarly, rs5751876 was evaluated in the pathogenosis of vasovagal syncope however no association with head-up tilt table test was reported [17]. Also homozygous CC carriers of rs2298383 were shown to have increased expression of ADORA2A receptor mRNA in brain and lymphoblasts [15].

Zhai et al [18] recently described an association between one common single nucleotide polymporphism (SNP) of ADORA2A (rs4822489) and the severity of congestive heart failure after adjustment for traditional risk factors (OR 1.912, 95\% CI $=1.029-3.550$ ) in a northern Chinese population. We did not genotype this particular variant, but we have observed a higher prevalence of congestive heart failure in carriers of the minor allele variant for rs2298383, rs3761422, rs2267076, and rs5751876. Further studies may be indicated to examine the influence of ADORA2A variants in congestive heart failure pathogenesis and phenotypes.

We hypothesized that 5 of the common $\mathrm{A} 2 \mathrm{~A}$ receptor variants that have been associated with variability in response to adenosine stimulation in non-cardiac phenotypes, would affect hemodynamic response to regadenoson, and possibly influence tolerability and detection of ischemia as defined by summed difference scores during regadenoson nuclear perfusion testing,. However none of the variants were associated with significant differences in reported adverse symptoms, nor significant differences in baseline and post regadenoson hemodynamic variables. Similarly, we could not document a significant 
difference in ischemia detected by nuclear perfusion imaging among genotypes. This indicates a low likelihood that the ADORA2A variants studied would be associated with a dampened vasodilator response to regadenoson or decrease in observed perfusion defects seen on nuclear testing. The finding of higher baseline perfusion defects (SRS) in carriers of rs2267076 and rs3761422 could be due to chance, or less likely be an indicator of higher prevalence of chronic ischemic heart disease in carriers of the variant, however there has been no prior data to suggest this. Small fixed perfusion defects are commonly observed with soft tissue attenuation artefacts particularly in obese individuals, which could have contributed to a small difference in SRS. This would be supported by higher body mass index seen in carriers of minor allele variants of rs2267076 and rs3761422 (Table 2).

\section{Conclusion}

Our findings suggest that there are no significant differences in hemodynamic response and side effects of regadenoson administration among genotypes of the five ADORA2A variants studied (rs5751862, rs229838, rs3761422, 2267076 and rs5751876). In addition, no significant difference in myocardial ischemia was detected on nuclear stress perfusion imaging with regadenoson among ADORA2A variants studied, indicating that these variants are unlikely to significantly affect regadenoson cardiac stress testing. 


\section{References}

1. Al Jaroudi W, Iskandrian AE. Regadenoson: A New Myocardial Stress Agent. Journal of the American College of Cardiology 54(13), 1123-1130 (2009).

2. Cerqueira MD, Nguyen P, Staehr P, Underwood SR, Iskandrian AE. Effects of Age, Gender, Obesity, and Diabetes on the Efficacy and Safety of the Selective A2A Agonist Regadenoson Versus Adenosine in Myocardial Perfusion Imaging: Integrated ADVANCE-MPI Trial Results. JACC: Cardiovascular Imaging 1(3), 307-316 (2008).

3. Bitar A, Mastouri R, Kreutz RP. Caffeine Consumption and Heart Rate and Blood Pressure Response to Regadenoson. PLoS One 10(6), e0130487 (2015).

4. Saab R ZA, Mastouri R, Skaar Tc, Philips S, Kreutz Rp. AMPD1 polymorphism and response to regadenoson. Pharmacogenomics 16(16), 1807-1815 (2015).

5. Molero Y, Gumpert C, Serlachius E et al. A study of the possible association between adenosine A2A receptor gene polymorphisms and attention-deficit hyperactivity disorder traits. Genes, Brain and Behavior 12(3), 305-310 (2013).

6. Turčin A DV, Porcelli S, Serretti a, Plesničar Bk. Adenosine Hypothesis of Antipsychotic Drugs Revisited: Pharmacogenomics Variation in Nonacute Schizophrenia. OMICS 20(5), 283-289 (2016).

7. Janik P BM, Safranow K, Żekanowski C. Association of ADORA1 rs2228079 and ADORA2A rs5751876 Polymorphisms with Gilles de la Tourette Syndrome in the Polish Population. PLoS One 10(8), e0136754 (2015).

8. Rogers Pj HC, Heatherley Sv, Mullings El, Maxfield Pj, Evershed Rp, Deckert J, Nutt Dj. Association of the anxiogenic and alerting effects of caffeine with ADORA2A and ADORA1 polymorphisms and habitual level of caffeine consumption. Neuropsychopharmacology 35(9), 1973-1983 (2010).

9. Hohoff C ME, Heatherley Sv, Freitag Cm, Neumann Lc, Domschke K, Krakowitzky P, Rothermundt M, Keck Me, Erhardt a, Unschuld Pg, Jacob C, Fritze J, Bandelow B, Maier W, Holsboer F, Rogers Pj, Deckert J. Adenosine A(2A) receptor gene: evidence for association of risk variants with panic disorder and anxious personality. $J$ Psychiatr Res 44(14), 930-937 (2010).

10. Rieck M S-SA, Callegari-Jacques Sm, Altmann V, Schneider Medeiros M, Rieder Cr, Hutz Mh. Is there a role for ADORA2A polymorphisms in levodopa-induced dyskinesia in Parkinson's disease patients? Pharmacogenomics 16(6), 573-582 (2015).

11. Trägårdh E HB, Knuuti J, Flotats a, Kaufmann Pa, Kitsiou a, Hacker M, Verberne Hj, Edenbrandt L, Delgado V, Donal E, Edvardsen T, Galderisi M, Habib G, Lancellotti P, Nieman K, Rosenhek R; Eacvi, Agostini D, Gimelli a, Lindner O, Slart R, Ubleis C; Eanm. Reporting nuclear cardiology: a joint position paper by the European Association of Nuclear Medicine (EANM) and the European Association of Cardiovascular Imaging (EACVI). Eur Heart J Cardiovasc Imaging 16(3), 272-279 (2015).

12. Labazi H TB, Zhou Z, Mustafa Sj. Enhanced A2A adenosine receptor-mediated increase in coronary flow in type I diabetic mice. J Mol Cell Cardiol 90 30-37 (2016;).

13. Rivkees SA, Wendler CC. Regulation of Cardiovascular Development by Adenosine and Adenosine-Mediated Embryo Protection. Arteriosclerosis, Thrombosis, and Vascular Biology 32(4), 851-855 (2012). 
14. Alsene K, Deckert J, Sand P, De Wit H. Association Between A2a Receptor Gene Polymorphisms and Caffeine-Induced Anxiety. Neuropsychopharmacology 28(9), 1694-1702 (2003).

15. Shinohara M, Saitoh M, Nishizawa D et al. ADORA2A polymorphism predisposes children to encephalopathy with febrile status epilepticus. Neurology 80(17), 15711576 (2013).

16. Charles BA, Conley YP, Chen G et al. Variants of the Adenosine A(2A) Receptor Gene Are Protective against Proliferative Diabetic Retinopathy in Patients with Type 1 Diabetes. Ophthalmic Research 46(1), 1-8 (2011).

17. Mitro P, Habalova V, Evin L et al. Gene Polymorphism of the Adenosine A2a Receptor in Patients with Vasovagal Syncope. Pacing and Clinical Electrophysiology doi:10.1111/pace.12806 n/a-n/a (2016).

18. Zhai Y-J, Liu P, He H-R et al. The Association of ADORA2A and ADORA2B Polymorphisms with the Risk and Severity of Chronic Heart Failure: A Case-Control Study of a Northern Chinese Population. International Journal of Molecular Sciences 16(2), 2732-2746 (2015). 
Table 1. Hardy-Weinberg Equilibrium

\begin{tabular}{|c|c|c|c|c|c|}
\hline \multirow{2}{*}{ A2A variants } & \multicolumn{3}{|c|}{ n (Alleles) } & $\begin{array}{l}\text { Minor Allele Carriers } \\
\text { (Minor Allele } \\
\text { Frequency) }\end{array}$ & P-value \\
\hline rs5751862 & $70(\mathrm{GG})$ & $160(\mathrm{AG})$ & $109(\mathrm{AA})$ & $230 / 339(0.44)$ & 0.42 \\
\hline rs2298383 & $84(\mathrm{CC})$ & $184(\mathrm{CT})$ & $87(\mathrm{TT})$ & $268 / 355(0.5)$ & 0.7 \\
\hline rs3761422 & $126(\mathrm{CC})$ & $177(\mathrm{CT})$ & $54(\mathrm{TT})$ & $231 / 357(0.4)$ & 0.53 \\
\hline rs2267076 & $142(\mathrm{CC})$ & $167(\mathrm{CT})$ & $46(\mathrm{TT})$ & $213 / 355(0.36)$ & 0.78 \\
\hline rs5751876 & $72(\mathrm{TT})$ & $185(\mathrm{CT})$ & $99(\mathrm{CC})$ & $257 / 356(0.46)$ & 0.39 \\
\hline
\end{tabular}


Table 2. Clinical Variables

\begin{tabular}{|c|c|c|c|c|c|c|c|c|c|c|c|c|c|c|c|c|c|c|c|c|}
\hline \multirow[t]{2}{*}{ A2A variants } & \multicolumn{4}{|c|}{ rs5751862 } & \multicolumn{4}{|c|}{ rs2298383 } & \multicolumn{4}{|c|}{ rs3761422 } & \multicolumn{4}{|c|}{ rs2267076 } & \multicolumn{4}{|c|}{ rs5751876 } \\
\hline & GG & AG & GG & $\begin{array}{l}\mathrm{p}- \\
\text { valu } \\
\mathrm{e}\end{array}$ & $\mathrm{CC}$ & CT & TT & $\begin{array}{l}\mathrm{p} \text { - } \\
\text { value }\end{array}$ & $\mathrm{CC}$ & CT & $\mathrm{TT}$ & $\begin{array}{l}\mathrm{p} \text { - } \\
\text { value }\end{array}$ & $\mathrm{CC}$ & $\mathrm{CT}$ & TT & $\begin{array}{l}p \text { - } \\
\text { value }\end{array}$ & $\mathrm{TT}$ & $\mathrm{CT}$ & $\mathrm{CC}$ & $\begin{array}{l}\mathrm{p}- \\
\text { value }\end{array}$ \\
\hline Age (years) & $\begin{array}{l}60.1 \pm 10 \\
.9\end{array}$ & $\begin{array}{l}59.1 \pm \\
11\end{array}$ & $\begin{array}{l}57.9 \\
\pm 10.5\end{array}$ & 0.38 & $\begin{array}{l}58.6 \pm 1 \\
0.4\end{array}$ & $59 \pm 11$ & $\begin{array}{l}59.8 \pm \\
10.9\end{array}$ & 0.76 & $\begin{array}{l}59.3 \pm 1 \\
0.7\end{array}$ & $\begin{array}{l}59 \pm \\
10.8\end{array}$ & $\begin{array}{l}58.5 \pm \\
10.4\end{array}$ & 0.89 & $\begin{array}{l}59.0 \pm 1 \\
0.8\end{array}$ & $\begin{array}{l}58.8 \pm \\
11\end{array}$ & $\begin{array}{l}59.2 \\
\pm 10\end{array}$ & 0.96 & $\begin{array}{l}58.6 \pm \\
10.3\end{array}$ & $\begin{array}{l}58.9 \pm 10 . \\
8\end{array}$ & $\begin{array}{l}59.4 \pm \\
11\end{array}$ & 0.88 \\
\hline Males & $\begin{array}{l}26 / 70 \\
(37 \%)\end{array}$ & $\begin{array}{l}76 / 160 \\
(48 \%)\end{array}$ & $\begin{array}{l}53 / 109 \\
(49 \%)\end{array}$ & & $\begin{array}{l}36 / 84 \\
(43 \%) \\
\end{array}$ & $\begin{array}{l}89 / 184 \\
(48 \%)\end{array}$ & $\begin{array}{l}39 / 87 \\
(45 \%)\end{array}$ & & $\begin{array}{l}59 / 126 \\
(47 \%)\end{array}$ & $\begin{array}{l}83 / 177 \\
(47 \%)\end{array}$ & $\begin{array}{l}22 / 54 \\
(41 \%)\end{array}$ & & $\begin{array}{l}64 / 142 \\
(45 \%)\end{array}$ & $\begin{array}{l}82 / 167 \\
(49 \%)\end{array}$ & $\begin{array}{l}18 / 46 \\
(39 \%)\end{array}$ & & $\begin{array}{l}32 / 72 \\
(44 \%) \\
\end{array}$ & $\begin{array}{l}83 / 185 \\
(45 \%)\end{array}$ & $\begin{array}{l}46 / 99 \\
(46 \%)\end{array}$ & \\
\hline Females & $\begin{array}{l}44 / 70 \\
(63 \%) \\
\end{array}$ & $\begin{array}{l}84 / 160 \\
(52 \%)\end{array}$ & $\begin{array}{l}56 / 109 \\
(51 \%)\end{array}$ & 0.27 & $\begin{array}{l}48 / 84 \\
(57 \%) \\
\end{array}$ & $\begin{array}{l}95 / 184 \\
(52 \%)\end{array}$ & $\begin{array}{l}48 / 87 \\
(55 \%) \\
\end{array}$ & 0.67 & $\begin{array}{l}67 / 126 \\
(53 \%) \\
\end{array}$ & $\begin{array}{l}94 / 177 \\
(53 \%)\end{array}$ & $\begin{array}{l}32 / 54 \\
(59 \%) \\
\end{array}$ & 0.71 & $\begin{array}{l}78 / 142 \\
(55 \%)\end{array}$ & $\begin{array}{l}85 / 167 \\
(51 \%)\end{array}$ & $\begin{array}{l}28 / 46 \\
(61 \%) \\
\end{array}$ & 0.46 & $\begin{array}{l}40 / 72 \\
(56 \%) \\
\end{array}$ & $\begin{array}{l}102 / 185 \\
(55 \%)\end{array}$ & $\begin{array}{l}53 / 99 \\
(54 \%) \\
\end{array}$ & 0.96 \\
\hline Caucasian & $\begin{array}{l}55 / 70 \\
(79 \%) \\
\end{array}$ & $\begin{array}{l}126 / 160 \\
(79 \%)\end{array}$ & $\begin{array}{l}61 / 109 \\
(56 \%)\end{array}$ & $\begin{array}{l}<0.0 \\
01\end{array}$ & $\begin{array}{l}46 / 84 \\
(55 \%) \\
\end{array}$ & $\begin{array}{l}134 / 184 \\
(73 \%)\end{array}$ & $\begin{array}{l}74 / 87 \\
(85 \%) \\
\end{array}$ & $\begin{array}{l}<0.0 \\
01\end{array}$ & $\begin{array}{l}85 / 126 \\
(68 \%) \\
\end{array}$ & $\begin{array}{l}129 / 17 \\
7 \\
(73 \%)\end{array}$ & $\begin{array}{l}41 / 54 \\
(76 \%)\end{array}$ & 0.51 & $\begin{array}{l}99 / 142 \\
(70 \%)\end{array}$ & $\begin{array}{l}120 / 16 \\
7 \\
(72 \%)\end{array}$ & $\begin{array}{l}36 / 46 \\
(78 \%)\end{array}$ & 0.53 & $\begin{array}{l}46 / 72 \\
(64 \%)\end{array}$ & $\begin{array}{l}131 / 185 \\
(71 \%)\end{array}$ & $\begin{array}{l}79 / 99 \\
(79 \%)\end{array}$ & 0.065 \\
\hline African American & $\begin{array}{l}13 / 70 \\
(19 \%)\end{array}$ & $\begin{array}{l}33 / 160 \\
(21 \%)\end{array}$ & $\begin{array}{l}45 / 109 \\
(41 \%)\end{array}$ & $\begin{array}{l}<0.0 \\
01\end{array}$ & $\begin{array}{l}34 / 84 \\
(41 \%)\end{array}$ & $\begin{array}{l}48 / 184 \\
(26 \%)\end{array}$ & $\begin{array}{l}13 / 87 \\
(15 \%)\end{array}$ & 0.001 & $\begin{array}{l}41 / 126 \\
(33 \%)\end{array}$ & $\begin{array}{l}44 / 177 \\
(25 \%)\end{array}$ & $\begin{array}{l}10 / 54 \\
(19 \%)\end{array}$ & 0.11 & $\begin{array}{l}43 / 142 \\
(30 \%)\end{array}$ & $\begin{array}{l}44 / 167 \\
(26 \%)\end{array}$ & $\begin{array}{l}7 / 46 \\
(15 \%)\end{array}$ & 0.13 & $\begin{array}{l}22 / 72 \\
(31 \%)\end{array}$ & $\begin{array}{l}52 / 185 \\
(28 \%)\end{array}$ & $\begin{array}{l}20 / 99 \\
(20 \%)\end{array}$ & 0.24 \\
\hline & $20 / 70$ & $53 / 160$ & $34 / 109$ & & $27 / 84$ & $53 / 184$ & $27 / 86$ & & $38 / 126$ & $52 / 176$ & $18 / 54$ & & $44 / 141$ & $47 / 166$ & $17 / 46$ & & $23 / 72$ & $56 / 184$ & $30 / 98$ & \\
\hline $\begin{array}{l}\text { Smoking } \\
\text { Body Mass Index }\end{array}$ & $\frac{(29 \%)}{34.3 \pm}$ & $\frac{(33 \%)}{34.3 \pm}$ & $\frac{(31 \%)}{36 \pm}$ & 0.8 & $\frac{(32 \%)}{36 \pm}$ & $\frac{(29 \%)}{35.1 \pm}$ & $\frac{(31 \%)}{32.5 \pm}$ & 0.8 & $\frac{(30 \%)}{33.2 \pm}$ & $\frac{(30 \%)}{35.7 \pm}$ & $\begin{array}{l}(33 \%) \\
35.4 \pm\end{array}$ & 0.87 & $\frac{(31 \%)}{33.3 \pm}$ & $\frac{(28 \%)}{35.6 \pm}$ & $\begin{array}{l}(37 \%) \\
35.6\end{array}$ & 0.52 & $\frac{(32 \%)}{35.6 .}$ & $(30 \%)$ & $\frac{(31 \%)}{32.7 \pm}$ & 0.97 \\
\hline$\frac{\left(\mathrm{kg} / \mathrm{m}^{2}\right)}{\mathrm{kngmon}}$ & 7.8 & 8.2 & 10.1 & 0.25 & 9.8 & 8.7 & 7.8 & 0.019 & 7.6 & 9.6 & 8.3 & 0.045 & 7.9 & 9.6 & \pm 8 & 0.07 & $6 \pm 8.9$ & $35.5 \pm 9$ & 7.9 & 0.025 \\
\hline $\begin{array}{l}\text { Known Coronary } \\
\text { Disease }\end{array}$ & $\begin{array}{l}18 / 70 \\
(26 \%)\end{array}$ & $\begin{array}{l}52 / 160 \\
(33 \%)\end{array}$ & $\begin{array}{l}27 / 109 \\
(25 \%)\end{array}$ & 0.32 & $\begin{array}{l}17 / 84 \\
(20 \%)\end{array}$ & $\begin{array}{l}58 / 184 \\
(32 \%)\end{array}$ & $\begin{array}{l}27 / 87 \\
(31 \%)\end{array}$ & 0.14 & $\begin{array}{l}38 / 126 \\
(30 \%)\end{array}$ & $\begin{array}{l}58 / 177 \\
(33 \%)\end{array}$ & $\begin{array}{l}9 / 54 \\
(17 \%)\end{array}$ & 0.07 & $\begin{array}{l}43 / 142 \\
(30 \%)\end{array}$ & $\begin{array}{l}55 / 167 \\
(33 \%)\end{array}$ & $\begin{array}{l}6 / 46 \\
(13 \%)\end{array}$ & 0.03 & $\begin{array}{l}16 / 72 \\
(22 \%)\end{array}$ & $\begin{array}{l}56 / 185 \\
(30 \%)\end{array}$ & $\begin{array}{l}29 / 99 \\
(29 \%)\end{array}$ & 0.31 \\
\hline & $16 / 70$ & $38 / 160$ & $18 / 109$ & & $15 / 84$ & $42 / 184$ & $20 / 87$ & & $23 / 126$ & $44 / 177$ & $10 / 54$ & & $26 / 142$ & $44 / 167$ & $6 / 46$ & & $14 / 72$ & $44 / 185$ & $20 / 99$ & \\
\hline$\frac{\text { History of stents }}{\text { CCoronary Artery }}$ & $(23 \%)$ & $(24 \%)$ & (17\%) & 0.34 & $(18 \%)$ & $(23 \%)$ & $(23 \%)$ & 0.62 & $(18 \%)$ & $(25 \%)$ & $(19 \%)$ & 0.33 & $(18 \%)$ & $(26 \%)$ & $(13 \%)$ & 0.08 & $(19 \%)$ & $(24 \%)$ & $(20 \%)$ & 0.67 \\
\hline $\begin{array}{l}\text { Coronary Artery } \\
\text { Bypass Graft } \\
\text { Surgery }\end{array}$ & $\begin{array}{l}6 / 70 \\
(9 \%)\end{array}$ & $\begin{array}{l}12 / 160 \\
(8 \%) \\
\end{array}$ & $\begin{array}{l}11 / 109 \\
(10 \%)\end{array}$ & 0.76 & $\begin{array}{l}10 / 84 \\
(12 \%)\end{array}$ & $\begin{array}{l}\begin{array}{l}14 / 184 \\
(8 \%)\end{array} \\
\end{array}$ & $\begin{array}{l}8 / 87 \\
(9 \%) \\
\end{array}$ & 0.52 & $\begin{array}{l}13 / 126 \\
(10 \%)\end{array}$ & $\begin{array}{l}15 / 177 \\
(8 \%)\end{array}$ & $\begin{array}{l}6 / 54 \\
(11 \%)\end{array}$ & 0.79 & $\begin{array}{l}14 / 142 \\
(10 \%)\end{array}$ & $\begin{array}{l}14 / 167 \\
(8 \%)\end{array}$ & $\begin{array}{l}5 / 46 \\
(11 \%) \\
\end{array}$ & 0.84 & $\begin{array}{l}9 / 72 \\
(13 \%) \\
\end{array}$ & $\begin{array}{l}14 / 185 \\
(76 \%)\end{array}$ & $\begin{array}{l}10 / 99 \\
(\%)\end{array}$ & 0.45 \\
\hline $\begin{array}{l}\text { Prior Myocardial } \\
\text { Infarction }\end{array}$ & $\begin{array}{l}10 / 70 \\
(14 \%)\end{array}$ & $\begin{array}{l}36 / 160 \\
(23 \%)\end{array}$ & $\begin{array}{l}23 / 109 \\
(21 \%)\end{array}$ & 0.35 & $\begin{array}{l}16 / 84 \\
(19 \%)\end{array}$ & $\begin{array}{l}36 / 184 \\
(20 \%)\end{array}$ & $\begin{array}{l}21 / 87 \\
(24 \%)\end{array}$ & 0.63 & $\begin{array}{l}26 / 126 \\
(21 \%)\end{array}$ & $\begin{array}{l}39 / 177 \\
(22 \%)\end{array}$ & $\begin{array}{l}10 / 54 \\
(19 \%)\end{array}$ & 0.85 & $\begin{array}{l}30 / 142 \\
(21 \%)\end{array}$ & $\begin{array}{l}36 / 167 \\
(22 \%)\end{array}$ & $\begin{array}{l}8 / 46 \\
(17 \%)\end{array}$ & 0.82 & $\begin{array}{l}15 / 72 \\
(21 \%)\end{array}$ & $\begin{array}{l}38 / 185 \\
(18 \%)\end{array}$ & $\begin{array}{l}22 / 99 \\
(22 \%)\end{array}$ & 0.95 \\
\hline Congestive Heart & $3 / 70$ & $13 / 160$ & $16 / 109$ & & $13 / 84$ & $18 / 184$ & $2 / 87$ & & $5 / 126$ & $19 / 177$ & $10 / 54$ & & $8 / 142$ & $18 / 167$ & $8 / 46$ & & $12 / 72$ & $18 / 185$ & $4 / 99$ & \\
\hline & & $(8 \%)$ & $(15 \%)$ & 0.05 & $(15 \%)$ & $(10 \%)$ & $(2 \%)$ & 0.012 & $(4 \%)$ & $(11 \%)$ & $(19 \%)$ & 0.007 & $(6 \%)$ & $(11 \%)$ & $(17 \%)$ & 0.048 & $(17 \%)$ & $(10 \%)$ & $(4 \%)$ & 0.021 \\
\hline Diabetes & $\begin{array}{l}27 / 70 \\
(39 \%)\end{array}$ & $\begin{array}{l}74 / 160 \\
(46 \%)\end{array}$ & $\begin{array}{l}43 / 109 \\
(39 \%)\end{array}$ & 0.41 & $\begin{array}{l}34 / 84 \\
(41 \%)\end{array}$ & $\begin{array}{l}83 / 184 \\
(45 \%)\end{array}$ & $\begin{array}{l}31 / 87 \\
(36 \%)\end{array}$ & 0.33 & $\begin{array}{l}53 / 126 \\
(42 \%)\end{array}$ & $\begin{array}{l}73 / 177 \\
(41 \%)\end{array}$ & $\begin{array}{l}25 / 54 \\
(46 \%)\end{array}$ & 0.8 & $\begin{array}{l}60 / 142 \\
(42 \%)\end{array}$ & $\begin{array}{l}67 / 167 \\
(40 \%)\end{array}$ & $\begin{array}{l}22 / 46 \\
(48 \%)\end{array}$ & 0.64 & $\begin{array}{l}32 / 72 \\
(44 \%)\end{array}$ & $\begin{array}{l}79 / 185 \\
(43 \%)\end{array}$ & $\begin{array}{l}38 / 99 \\
(38 \%)\end{array}$ & 0.69 \\
\hline Hypertension & $\begin{array}{l}54 / 70 \\
(77 \%) \\
\end{array}$ & $\begin{array}{l}135 / 160 \\
(84 \%)\end{array}$ & $\begin{array}{l}81 / 109 \\
(74 \%)\end{array}$ & 0.11 & $\begin{array}{l}64 / 84 \\
(76 \%)\end{array}$ & $\begin{array}{l}153 / 184 \\
(83 \%)\end{array}$ & $\begin{array}{l}61 / 87 \\
(70 \% \\
\end{array}$ & 0.045 & $\begin{array}{l}94 / 126 \\
(75 \%)\end{array}$ & $\begin{array}{l}146 / 17 \\
7 \\
(82 \%)\end{array}$ & $\begin{array}{l}41 / 54 \\
(76 \%) \\
\end{array}$ & 0.22 & $\begin{array}{l}108 / 14 \\
2 \\
(76 \%)\end{array}$ & $\begin{array}{l}138 / 16 \\
7 \\
(83 \%)\end{array}$ & $\begin{array}{l}35 / 46 \\
(76 \%)\end{array}$ & 0.31 & $\begin{array}{l}55 / 72 \\
(76 \%) \\
\end{array}$ & $\begin{array}{l}155 / 185 \\
(84 \%)\end{array}$ & $\begin{array}{l}70 / 99 \\
(71 \%)\end{array}$ & 0.033 \\
\hline $\begin{array}{l}\text { Atrial Fibrillation or } \\
\text { Flutter }\end{array}$ & $\begin{array}{l}7 / 70 \\
(10 \%)\end{array}$ & $\begin{array}{l}22 / 160 \\
(14 \%)\end{array}$ & $\begin{array}{l}16 / 109 \\
(15 \%)\end{array}$ & 0.65 & $\begin{array}{l}9 / 84 \\
(11 \%)\end{array}$ & $\begin{array}{l}23 / 184 \\
(13 \%)\end{array}$ & $\begin{array}{l}13 / 87 \\
(15 \%)\end{array}$ & 0.70 & $\begin{array}{l}17 / 126 \\
(14 \%)\end{array}$ & $\begin{array}{l}24 / 177 \\
(14 \%)\end{array}$ & $\begin{array}{l}5 / 54 \\
(9 \%)\end{array}$ & 0.69 & $\begin{array}{l}20 / 142 \\
(14 \%)\end{array}$ & $\begin{array}{l}21 / 167 \\
(13 \%)\end{array}$ & $\begin{array}{l}5 / 46 \\
(11 \%)\end{array}$ & 0.84 & $\begin{array}{l}6 / 72 \\
(8 \%)\end{array}$ & $\begin{array}{l}27 / 185 \\
(15 \%)\end{array}$ & $\begin{array}{l}13 / 99 \\
(13 \%)\end{array}$ & 0.4 \\
\hline $\begin{array}{l}\text { COPD or } \\
\text { Emphysema }\end{array}$ & $18 / 70$ & $28 / 160$ & $25 / 109$ & 031 & $25 / 84$ & $31 / 184$ & $18 / 87$ & 0054 & $20 / 126$ & $36 / 177$ & $17 / 54$ & مी6 & $29 / 142$ & $29 / 167$ & $16 / 46$ & 0026 & $19 / 72$ & $37 / 185$ & $19 / 99$ & \\
\hline & $12 / 70$ & $32 / 160$ & $\frac{25 \%)}{24 / 109}$ & & $\frac{150 \%)}{20 / 84}$ & $33 / 184$ & $\frac{(21 / 0)}{18 / 87}$ & & $\frac{1(10 \%)}{26 / 126}$ & $\frac{(20 \%)}{33 / 177}$ & $\frac{111 \%)}{12 / 54}$ & & $\frac{20 \%)}{29 / 142}$ & $32 / 167$ & $\frac{(35 \%)}{12 / 46}$ & & $\frac{(26 \% / 0)}{16 / 72}$ & $38 / 185$ & $\frac{19 \%)}{19 / 99}$ & 0.40 \\
\hline Asthma & (17\%) & $(20 \%$ & $\begin{array}{l}(22 \%) \\
\end{array}$ & 0.73 & $(24 \%)$ & $\begin{array}{l}(18 \%) \\
(18)\end{array}$ & $(21 \%)$ & 0.53 & $\begin{array}{l}(21 \%) \\
\end{array}$ & $(19 \%)$ & (22\%) & 0.82 & & $\begin{array}{l}(19 \%) \\
(19 \%)\end{array}$ & $(26 \%)$ & 0.59 & $(22 \%)$ & $\begin{array}{l}(21 \%) \\
\text { (21) }\end{array}$ & $(19 \%)$ & 0.89 \\
\hline Stroke & $\begin{array}{l}3 / 70 \\
(4 \%) \\
\end{array}$ & $\begin{array}{l}20 / 160 \\
(13 \%)\end{array}$ & $\begin{array}{l}\begin{array}{l}7 / 109 \\
(6 \%)\end{array} \\
\end{array}$ & 0.07 & $\begin{array}{l}9 / 84 \\
(11 \%) \\
\end{array}$ & $\begin{array}{l}17 / 184 \\
(9 \%) \\
\end{array}$ & $\begin{array}{l}6 / 87 \\
(7 \%) \\
\end{array}$ & 0.68 & $\begin{array}{l}\begin{array}{l}11 / 126 \\
(9 \%)\end{array} \\
\end{array}$ & $\begin{array}{l}17 / 177 \\
(10 \%)\end{array}$ & $\begin{array}{l}4 / 54 \\
(7 \%)\end{array}$ & 0.88 & $\begin{array}{l}12 / 142 \\
(9 \%)\end{array}$ & $\begin{array}{l}15 / 167 \\
(9 \%)\end{array}$ & $\begin{array}{l}3 / 46 \\
(7 \%)\end{array}$ & 0.87 & $\begin{array}{l}5 / 72 \\
(7 \%)\end{array}$ & $\begin{array}{l}20 / 185 \\
(11 \%)\end{array}$ & $\begin{array}{l}7 / 99 \\
(7 \%)\end{array}$ & 0.46 \\
\hline Cancer & $\begin{array}{l}5 / 70 \\
(7 \%)\end{array}$ & $\begin{array}{l}17 / 160 \\
(11 \%)\end{array}$ & $\begin{array}{l}11 / 109 \\
(10 \%)\end{array}$ & 0.71 & $\begin{array}{l}9 / 84 \\
(11 \%)\end{array}$ & $\begin{array}{l}15 / 184 \\
(8 \%)\end{array}$ & $\begin{array}{l}11 / 87 \\
(13 \%)\end{array}$ & 0.49 & $\begin{array}{l}16 / 126 \\
(13 \%)\end{array}$ & $\begin{array}{l}15 / 177 \\
(8 \%)\end{array}$ & $\begin{array}{l}5 / 54 \\
(9 \%)\end{array}$ & 0.47 & $\begin{array}{l}17 / 142 \\
(12 \%)\end{array}$ & $\begin{array}{l}14 / 167 \\
(8 \%)\end{array}$ & $\begin{array}{l}5 / 46 \\
(11 \%)\end{array}$ & 0.57 & $\begin{array}{l}7 / 72 \\
(10 \%)\end{array}$ & $\begin{array}{l}17 / 185 \\
(9 \%)\end{array}$ & $\begin{array}{l}11 / 99 \\
(11 \%)\end{array}$ & 0.87 \\
\hline Hyperlipidemia & $\begin{array}{l}49 / 70 \\
(70 \%)\end{array}$ & $\begin{array}{l}109 / 160 \\
(68 \%)\end{array}$ & $\begin{array}{l}70 / 109 \\
(64 \%)\end{array}$ & 0.69 & $\begin{array}{l}55 / 84 \\
(66 \%)\end{array}$ & $\begin{array}{l}131 / 184 \\
(71 \%)\end{array}$ & $\begin{array}{l}54 / 87 \\
(62 \%)\end{array}$ & 0.29 & $\begin{array}{l}78 / 126 \\
(62 \%)\end{array}$ & $\begin{array}{l}103 / 17 \\
7 \\
(69 \%)\end{array}$ & $\begin{array}{l}40 / 54 \\
(74 \%)\end{array}$ & 0.2 & $\begin{array}{l}90 / 142 \\
(63 \%)\end{array}$ & $\begin{array}{l}113 / 16 \\
7 \\
(68 \%)\end{array}$ & $\begin{array}{l}37 / 46 \\
(80 \%)\end{array}$ & 0.1 & $\begin{array}{l}52 / 72 \\
(72 \%)\end{array}$ & $\begin{array}{l}129 / 185 \\
(70 \%\end{array}$ & $\begin{array}{l}61 / 99 \\
(62 \%)\end{array}$ & 0.26 \\
\hline $\begin{array}{l}\text { Peripheral Vascular } \\
\text { Disease }\end{array}$ & $\begin{array}{l}6 / 70 \\
(9 \%)\end{array}$ & $\begin{array}{l}18 / 160 \\
(11 \%)\end{array}$ & $\begin{array}{l}14 / 109 \\
(13 \%)- \\
-\end{array}$ & 0.68 & $\begin{array}{l}8 / 84 \\
(10 \%)\end{array}$ & $\begin{array}{l}20 / 184 \\
(11 \%)\end{array}$ & $\begin{array}{l}11 / 87 \\
(13 \%)\end{array}$ & 0.81 & $\begin{array}{l}16 / 126 \\
(13 \%)\end{array}$ & $\begin{array}{l}20 / 177 \\
(11 \%)\end{array}$ & $\begin{array}{l}4 / 54 \\
(7 \%)\end{array}$ & 0.59 & $\begin{array}{l}17 / 142 \\
(12 \%)\end{array}$ & $\begin{array}{l}20 / 167 \\
(12 \%)\end{array}$ & $\begin{array}{l}3 / 46 \\
(7 \%)\end{array}$ & 0.55 & $\begin{array}{l}5 / 72 \\
(7 \%)\end{array}$ & $\begin{array}{l}22 / 185 \\
(12 \%)\end{array}$ & $\begin{array}{l}13 / 99 \\
(13 \%)\end{array}$ & 0.41 \\
\hline Beta Blocker & $\begin{array}{l}31 / 70 \\
(44 \%) \\
\end{array}$ & $\begin{array}{l}76 / 160 \\
(48 \%)\end{array}$ & $\begin{array}{l}51 / 109 \\
(47 \%)\end{array}$ & 0.9 & $\begin{array}{l}37 / 84 \\
(44 \%)\end{array}$ & $\begin{array}{l}86 / 184 \\
(47 \%)\end{array}$ & $\begin{array}{l}44 / 87 \\
(51 \%) \\
\end{array}$ & 0.69 & $\begin{array}{l}61 / 126 \\
(48 \%) \\
\end{array}$ & $\begin{array}{l}84 / 177 \\
(47 \%)\end{array}$ & $\begin{array}{l}25 / 54 \\
(46 \%) \\
\end{array}$ & 0.99 & $\begin{array}{l}67 / 142 \\
(47 \%)\end{array}$ & $\begin{array}{l}80 / 167 \\
(48 \%)\end{array}$ & $\begin{array}{l}21 / 46 \\
(46 \%) \\
\end{array}$ & 0.96 & $\begin{array}{l}34 / 72 \\
(47 \%)\end{array}$ & $\begin{array}{l}87 / 185 \\
(47 \%)\end{array}$ & $\begin{array}{l}46 / 99 \\
(46 \%) \\
\end{array}$ & 0.99 \\
\hline $\begin{array}{l}\text { Calcium Channel } \\
\text { Blocker }\end{array}$ & $\begin{array}{l}17 / 70 \\
(24 \%)\end{array}$ & $\begin{array}{l}50 / 160 \\
(31 \%)\end{array}$ & $\begin{array}{l}31 / 109 \\
(28 \%)\end{array}$ & 0.56 & $\begin{array}{l}24 / 84 \\
(29 \%)\end{array}$ & $\begin{array}{l}55 / 184 \\
(30 \%)\end{array}$ & $\begin{array}{l}21 / 87 \\
(24 \%)\end{array}$ & 0.61 & $\begin{array}{l}32 / 126 \\
(25 \%)\end{array}$ & $\begin{array}{l}54 / 177 \\
(31 \%)\end{array}$ & $\begin{array}{l}14 / 54 \\
(26 \%)\end{array}$ & 0.58 & $\begin{array}{l}39 / 142 \\
(28 \%)\end{array}$ & $\begin{array}{l}48 / 167 \\
(29 \%)\end{array}$ & $\begin{array}{l}11 / 46 \\
(24 \%)\end{array}$ & 0.81 & $\begin{array}{l}21 / 72 \\
(29 \%)\end{array}$ & $\begin{array}{l}55 / 185 \\
(30 \%)\end{array}$ & $\begin{array}{l}24 / 99 \\
(24 \%)\end{array}$ & 0.6 \\
\hline ACEIARB & $\begin{array}{l}33 / 70 \\
(47 \%)\end{array}$ & $\begin{array}{l}73 / 160 \\
(46 \%)\end{array}$ & $\begin{array}{l}48 / 109 \\
(44 \%)\end{array}$ & 0.92 & $\begin{array}{l}41 / 84 \\
(49 \%)\end{array}$ & $\begin{array}{l}80 / 184 \\
(43 \%)\end{array}$ & $\begin{array}{l}38 / 87 \\
(44 \%)\end{array}$ & 0.7 & $\begin{array}{l}57 / 126 \\
(45 \%)\end{array}$ & $\begin{array}{l}79 / 177 \\
(45 \%)\end{array}$ & $\begin{array}{l}27 / 54 \\
(50 \%)\end{array}$ & 0.78 & $\begin{array}{l}61 / 142 \\
(43 \%)\end{array}$ & $\begin{array}{l}77 / 167 \\
(46 \%)\end{array}$ & $\begin{array}{l}23 / 46 \\
(50 \%)\end{array}$ & 0.68 & $\begin{array}{l}36 / 72 \\
(50 \%)\end{array}$ & $\begin{array}{l}87 / 185 \\
(47 \%)\end{array}$ & $\begin{array}{l}11 / 99 \\
(41 \%)\end{array}$ & 0.5 \\
\hline
\end{tabular}


Table 3. Symptoms during Regadenoson administration

\begin{tabular}{|c|c|c|c|c|c|c|c|}
\hline & & \multicolumn{3}{|c|}{$\geq 1$ Symptoms } & \multicolumn{3}{|c|}{$\geq 3$ Symptoms } \\
\hline & & $\begin{array}{l}\text { Percentage } \\
\text { (actual) }\end{array}$ & $\begin{array}{l}\text { Odds Ratio (95\% } \\
\mathrm{Cl})\end{array}$ & $\begin{array}{l}\mathrm{p}- \\
\text { value }\end{array}$ & $\begin{array}{l}\text { Percentage } \\
\text { (actual) }\end{array}$ & $\begin{array}{l}\text { Odds Ratio (95\% } \\
\mathrm{Cl})\end{array}$ & $\begin{array}{l}\mathrm{p}- \\
\text { value }\end{array}$ \\
\hline \multirow{2}{*}{$\begin{array}{c}\mathrm{rs} 575186 \\
2\end{array}$} & $\begin{array}{l}\text { Carriers Minor } \\
\text { Allele }\end{array}$ & $198 / 230(86 \%)$ & \multirow{2}{*}{$1.6(0.86-2.9)$} & \multirow[t]{2}{*}{0.14} & $53 / 230(23 \%)$ & \multirow{2}{*}{$1.3(0.75-2.34)$} & \multirow[t]{2}{*}{0.33} \\
\hline & Non Carriers & $87 / 109(80 \%)$ & & & $20 / 109(18 \%)$ & & \\
\hline \multirow{2}{*}{$\begin{array}{c}\text { rs229838 } \\
3\end{array}$} & $\begin{array}{l}\text { Carriers Minor } \\
\text { Allele }\end{array}$ & $226 / 268(84 \%)$ & \multirow{2}{*}{$1.03(0.5-2)$} & \multirow[t]{2}{*}{0.93} & $62 / 268(23 \%)$ & \multirow{2}{*}{$1.14(0.623-2.086)$} & \multirow[t]{2}{*}{0.2} \\
\hline & Non Carriers & $73 / 87(84 \%)$ & & & $18 / 87(21 \%)$ & & \\
\hline \multirow{2}{*}{$\begin{array}{c}\mathrm{rs} 376142 \\
2\end{array}$} & $\begin{array}{l}\text { Carriers Minor } \\
\text { Allele }\end{array}$ & $197 / 231(85 \%)$ & \multirow{2}{*}{$1.16(0.64-2.1)$} & \multirow{2}{*}{0.63} & $57 / 231(25 \%)$ & \multirow{2}{*}{$1.55(0.9-2.7)$} & \multirow{2}{*}{0.12} \\
\hline & Non Carriers & $105 / 126(83 \%)$ & & & $22 / 126(18 \%)$ & & \\
\hline \multirow{2}{*}{$\begin{array}{c}\text { rs } 226707 \\
6\end{array}$} & $\begin{array}{l}\text { Carriers Minor } \\
\text { Allele }\end{array}$ & $180 / 213(85 \%)$ & \multirow[t]{2}{*}{$1.05(0.6-1.9)$} & \multirow[t]{2}{*}{0.86} & $51 / 213(24 \%)$ & \multirow[t]{2}{*}{$1.18(0.71-2)$} & \multirow[t]{2}{*}{0.54} \\
\hline & Non Carriers & $119 / 142(84 \%)$ & & & $30 / 142(21 \%)$ & & \\
\hline \multirow{2}{*}{$\begin{array}{c}\text { rs575187 } \\
6\end{array}$} & $\begin{array}{l}\text { Carriers Minor } \\
\text { Allele }\end{array}$ & $217 / 257(84 \%)$ & \multirow{2}{*}{$0.97(0.51-1.8)$} & \multirow{2}{*}{0.92} & $62 / 257(24 \%)$ & \multirow[t]{2}{*}{$1.34(0.75-2.4)$} & \multirow[t]{2}{*}{0.32} \\
\hline & Non Carriers & $84 / 99(85 \%)$ & & & 19/99 (19\%) & & \\
\hline
\end{tabular}


Table 4. Hemodynamic Response to Regadenoson Administration and Nuclear

\section{Perfusion Imaging}

\begin{tabular}{|l|c|c|c|c|c|c|c|}
\hline \multicolumn{4}{|c|}{ rs5751862 } & \multicolumn{5}{c|}{ p values } \\
\hline & $(\mathrm{GG})$ & $(\mathrm{AG})$ & $(\mathrm{AA})$ & $\begin{array}{c}\text { GG VS } \\
\text { AG }\end{array}$ & $\begin{array}{c}\text { GG vs } \\
\text { AA }\end{array}$ & $\begin{array}{c}\text { AG vS } \\
\text { AA }\end{array}$ & $\begin{array}{c}\text { ANOV } \\
\text { A }\end{array}$ \\
\hline Baseline Heart Rate & $\begin{array}{c}69.5 \pm 13 . \\
5\end{array}$ & $\begin{array}{c}71.3 \pm 12 \\
6\end{array}$ & $72.7 \pm 13$ & 0.99 & 0.32 & 1.0 & 0.27 \\
\hline Heart Rate Change & $\begin{array}{c}29.7 \pm 11 . \\
1\end{array}$ & $\begin{array}{c}28.8 \pm 14 \\
9\end{array}$ & $29.5 \pm 13$ & 1.0 & 1.0 & 1.0 & 0.99 \\
\hline Baseline Systolic Blood Pressure & $\begin{array}{c}137 \pm 21 . \\
7\end{array}$ & $\begin{array}{c}136 \pm 22 . \\
8\end{array}$ & $\begin{array}{c}136 \pm 20 \\
7\end{array}$ & 1.0 & 1.0 & 1.0 & 0.98 \\
\hline $\begin{array}{l}\text { Systolic Blood Pressure Change } \\
\text { 1min }\end{array}$ & $-2.8 \pm 22$ & $-2.8 \pm 16$ & $-1.6 \pm 15$ & 1.0 & 1.0 & 1.0 & 0.73 \\
\hline $\begin{array}{l}\text { Systolic Blood Pressure Change } \\
\text { 3min }\end{array}$ & $-0.6 \pm 18$ & $-3.2 \pm 20$ & $-2.5 \pm 19$ & 1.0 & 1.0 & 1.0 & 0.58 \\
\hline & & & & & & & \\
\hline Summed Stress Score (SSS) & $\begin{array}{c}1.56 \pm 3.4 \\
1\end{array}$ & $\begin{array}{c}2.57 \pm 5.2 \\
7\end{array}$ & $\begin{array}{c}3.29 \pm 5.6 \\
6\end{array}$ & 0.52 & 0.08 & 0.77 & 0.09 \\
\hline Summed Resting Score (SRS) & $\begin{array}{c}1.39 \pm 3.4 \\
7\end{array}$ & $\begin{array}{c}1.83 \pm 4.9 \\
8\end{array}$ & $\begin{array}{c}2.73 \pm 5.5 \\
1\end{array}$ & 1.0 & 0.25 & 0.45 & 0.18 \\
\hline Summed Difference Score (SDS) & $\begin{array}{c}0.57 \pm 1.6 \\
2\end{array}$ & $0.88 \pm 1.6$ & $\begin{array}{c}1.03 \pm 2.0 \\
9\end{array}$ & 0.67 & 0.3 & 1.0 & 0.25 \\
\hline
\end{tabular}

\begin{tabular}{|c|c|c|c|c|c|c|c|}
\hline \multicolumn{4}{|c|}{ rs2298383 } & \multicolumn{4}{|c|}{$p$ values } \\
\hline & $(\mathrm{CC})$ & (CT) & (TT) & $\begin{array}{c}\text { CC vs } \\
\text { CT }\end{array}$ & $\begin{array}{l}\text { CC vs } \\
\text { TT }\end{array}$ & $\begin{array}{c}\text { CT vs } \\
\text { TT }\end{array}$ & $\begin{array}{c}\text { ANOV } \\
\text { A }\end{array}$ \\
\hline Baseline Heart Rate & $\begin{array}{l}72.0 \pm 12 \\
7\end{array}$ & $\begin{array}{l}71.9 \pm 13 . \\
3\end{array}$ & $69.8 \pm 13$ & 1.0 & 0.78 & 0.65 & 0.41 \\
\hline Heart Rate Change & $\begin{array}{l}30.1 \pm 13 \\
8\end{array}$ & $\begin{array}{l}29.6 \pm 14 . \\
5\end{array}$ & $\begin{array}{l}29.6 \pm 10 . \\
7\end{array}$ & 1.0 & 1.0 & 1.0 & 0.95 \\
\hline Baseline Systolic Blood Pressure & $\begin{array}{l}138 \pm 19 . \\
5\end{array}$ & $\begin{array}{l}137 \pm 23 \\
1\end{array}$ & $134 \pm 21$ & 1.0 & 0.56 & 0.6 & 0.34 \\
\hline $\begin{array}{l}\text { Systolic Blood Pressure Change } \\
\text { 1min }\end{array}$ & $-3.6 \pm 15$ & $-2 \pm 16$ & $-2.4 \pm 22$ & 1.0 & 1.0 & 1.0 & 0.82 \\
\hline $\begin{array}{l}\text { Systolic Blood Pressure Change } \\
\text { 3min }\end{array}$ & $-1.6 \pm 20$ & $-1.6 \pm 19$ & $-4.9 \pm 17$ & 1.0 & 1.0 & 1.0 & 0.41 \\
\hline & & & & & & & \\
\hline Summed Stress Score (SSS) & $\begin{array}{l}2.80 \pm 5.4 \\
3\end{array}$ & $\begin{array}{l}3.02 \pm 5.3 \\
6\end{array}$ & $\begin{array}{l}2.00 \pm 4.0 \\
8\end{array}$ & 1.0 & 0.92 & 0.38 & 0.3 \\
\hline Summed Resting Score (SRS) & $\begin{array}{l}2.53 \pm 5.3 \\
5\end{array}$ & $2.17 \pm 5.1$ & $\begin{array}{l}1.49 \pm 3.7 \\
9\end{array}$ & 1.0 & 0.52 & 0.87 & 0.37 \\
\hline Summed Difference Score (SDS) & $\begin{array}{l}0.77 \pm 1.7 \\
1\end{array}$ & $\begin{array}{l}1.06 \pm 1.9 \\
6\end{array}$ & $\begin{array}{l}0.93 \pm 1.8 \\
7\end{array}$ & 0.77 & 1.0 & 1.0 & 0.45 \\
\hline
\end{tabular}

\begin{tabular}{|c|c|c|c|c|c|c|c|}
\hline \multicolumn{4}{|c|}{ rs3761422 } & \multicolumn{4}{|c|}{$p$ values } \\
\hline & (CC) & (CT) & (TT) & $\begin{array}{c}\text { CC vs } \\
\text { CT }\end{array}$ & $\begin{array}{c}\text { CC vs } \\
\text { TT }\end{array}$ & $\begin{array}{c}\text { CT vs } \\
\text { TT }\end{array}$ & $\begin{array}{c}\text { ANOV } \\
\text { A }\end{array}$ \\
\hline Baseline Heart Rate & $\begin{array}{l}69.9 \pm 12 \\
7\end{array}$ & $\begin{array}{l}72.0 \pm 13 . \\
6\end{array}$ & $\begin{array}{l}73.1 \pm 12 . \\
1\end{array}$ & 0.48 & 0.38 & 1.0 & 0.22 \\
\hline Heart Rate Change & $\begin{array}{l}29.6 \pm 10 \text {. } \\
7\end{array}$ & $\begin{array}{l}30.4 \pm 15 . \\
2\end{array}$ & $\begin{array}{l}27.8 \pm 13 . \\
3\end{array}$ & 1.0 & 1.0 & 0.69 & 0.48 \\
\hline Baseline Systolic Blood Pressure & $136 \pm 22$ & $\begin{array}{l}136 \pm 22 \\
1\end{array}$ & $\begin{array}{l}140 \pm 20 \\
8\end{array}$ & 1.0 & 0.85 & 1.0 & 0.59 \\
\hline
\end{tabular}




\begin{tabular}{|c|c|c|c|c|c|c|c|}
\hline $\begin{array}{l}\text { Systolic Blood Pressure Change } \\
\text { 1min }\end{array}$ & $-3.1 \pm 18$ & $-1.2 \pm 17$ & $-5 \pm 16$ & 1.0 & 1.0 & 0.53 & 0.38 \\
\hline $\begin{array}{l}\text { Systolic Blood Pressure Change } \\
\text { 3min }\end{array}$ & $-3.6 \pm 17$ & $-1.2 \pm 17$ & $-1.5 \pm 18$ & 1.0 & 1.0 & 1.0 & 0.74 \\
\hline Summed Stress Score (SSS) & $\begin{array}{l}1.85 \pm 3.6 \\
8\end{array}$ & $\begin{array}{l}3.17 \pm 5.6 \\
9\end{array}$ & $\begin{array}{l}3.24 \pm 5.8 \\
9\end{array}$ & 0.09 & 0.29 & 1.0 & 0.07 \\
\hline Summed Resting Score (SRS) & $\begin{array}{l}1.21 \pm 3.2 \\
7\end{array}$ & $\begin{array}{l}2.44 \pm 5.4 \\
8\end{array}$ & $\begin{array}{l}2.83 \pm 5.6 \\
4\end{array}$ & 0.1 & 0.13 & 1.0 & 0.047 \\
\hline Summed Difference Score (SDS) & $\begin{array}{l}0.91 \pm 1.8 \\
8\end{array}$ & $\begin{array}{l}0.95 \pm 1.8 \\
6\end{array}$ & $\begin{array}{l}0.92 \pm 1.8 \\
8\end{array}$ & 1.0 & 1.0 & 1.0 & 0.98 \\
\hline
\end{tabular}

\begin{tabular}{|c|c|c|c|c|c|c|c|}
\hline \multicolumn{4}{|c|}{ rs2267076 } & \multicolumn{4}{|c|}{ p values } \\
\hline & $(\mathrm{CC})$ & $(\mathrm{CT})$ & (TT) & $\begin{array}{c}\text { CC vs } \\
\text { CT }\end{array}$ & $\begin{array}{l}\text { CC vs } \\
\text { TT }\end{array}$ & $\begin{array}{l}\text { CT vs } \\
\text { TT }\end{array}$ & $\begin{array}{l}\text { ANOV } \\
\text { A }\end{array}$ \\
\hline Baseline Heart Rate & $70.1 \pm 13$ & $\begin{array}{l}72.4 \pm 13 . \\
5\end{array}$ & $\begin{array}{l}72.3 \pm 11 \text {. } \\
3\end{array}$ & 0.35 & 0.99 & 1.0 & 0.26 \\
\hline Heart Rate Change & $\begin{array}{l}30.2 \pm 11 \\
6\end{array}$ & $\begin{array}{l}30.0 \pm 14 \\
8\end{array}$ & $\begin{array}{l}28.2 \pm 13 \text {. } \\
5\end{array}$ & 1.0 & 1.0 & 1.0 & 0.66 \\
\hline Baseline Systolic Blood Pressure & $\begin{array}{l}135 \pm 21 \\
7\end{array}$ & $\begin{array}{l}137 \pm 22 \\
3\end{array}$ & $\begin{array}{l}138 \pm 21 \\
3\end{array}$ & 1.0 & 1.0 & 1.0 & 0.78 \\
\hline $\begin{array}{l}\text { Systolic Blood Pressure Change } \\
\text { 1min }\end{array}$ & $-2.8 \pm 19$ & $-1.5 \pm 17$ & $-4.5 \pm 16$ & 1.0 & 1.0 & 1.0 & 0.62 \\
\hline $\begin{array}{l}\text { Systolic Blood Pressure Change } \\
\text { 3min }\end{array}$ & $-3 \pm 18$ & $-2.3 \pm 20$ & $-1.7 \pm 19$ & 1.0 & 1.0 & 1.0 & 0.91 \\
\hline Summed Stress Score (SSS) & $\begin{array}{l}1.81 \pm 3.4 \\
9\end{array}$ & $\begin{array}{l}3.51 \pm 6.6 \\
9\end{array}$ & $\begin{array}{l}3.02 \pm 4.6 \\
8\end{array}$ & 0.02 & 0.56 & 1.0 & 0.024 \\
\hline Summed Resting Score (SRS) & $\begin{array}{l}1.16 \pm 3.1 \\
4\end{array}$ & $\begin{array}{l}2.81 \pm 6.3 \\
5\end{array}$ & $2.73 \pm 5.2$ & 0.02 & 0.24 & 1.0 & 0.018 \\
\hline Summed Difference Score (SDS) & $\begin{array}{l}0.85 \pm 1.7 \\
4\end{array}$ & $\begin{array}{l}0.94 \pm 1.8 \\
6\end{array}$ & $0.89 \pm 1.8$ & 1.0 & 1.0 & 1.0 & 0.92 \\
\hline
\end{tabular}

\begin{tabular}{|c|c|c|c|c|c|c|c|}
\hline \multicolumn{4}{|c|}{ rs5751876 } & \multicolumn{4}{|c|}{ p values } \\
\hline & $(\mathrm{TT})$ & (CT) & $(\mathrm{CC})$ & $\begin{array}{l}\text { TT vs } \\
\text { CT }\end{array}$ & $\begin{array}{l}\text { TT vs } \\
\text { CC }\end{array}$ & $\begin{array}{l}\text { CT vs } \\
\text { CC }\end{array}$ & $\begin{array}{l}\text { ANOV } \\
\text { A }\end{array}$ \\
\hline Baseline Heart Rate & $\begin{array}{l}71.5 \pm 12 \\
2\end{array}$ & $\begin{array}{l}72.3 \pm 13 \\
3\end{array}$ & $\begin{array}{l}69.5 \pm 13 . \\
2\end{array}$ & 1.0 & 0.92 & 0.25 & 0.22 \\
\hline Heart Rate Change & $\begin{array}{l}29.1 \pm 13 \\
8\end{array}$ & $\begin{array}{l}30.5 \pm 14 \text {. } \\
7\end{array}$ & $28.8 \pm 9.5$ & 1.0 & 1.0 & 0.94 & 0.55 \\
\hline Baseline Systolic Blood Pressure & $\begin{array}{l}140 \pm 20 \\
9\end{array}$ & $\begin{array}{l}136 \pm 22 \\
0\end{array}$ & $\begin{array}{l}134 \pm 22 \\
4\end{array}$ & 0.52 & 0.27 & 1.0 & 0.22 \\
\hline $\begin{array}{l}\text { Systolic Blood Pressure Change } \\
\text { 1min }\end{array}$ & $-4.6 \pm 15$ & $-1.2 \pm 17$ & $-2.2 \pm 21$ & 0.61 & 1.0 & 1.0 & 0.45 \\
\hline $\begin{array}{l}\text { Systolic Blood Pressure Change } \\
\text { 3min }\end{array}$ & $-2.8 \pm 18$ & $-1 \pm 20$ & $-4 \pm 16$ & 1.0 & 1.0 & 0.67 & 0.46 \\
\hline Summed Stress Score (SSS) & $3.28 \pm 5.8$ & $2.93 \pm 5.4$ & $\begin{array}{l}1.91 \pm 3.9 \\
1\end{array}$ & 1.0 & 0.26 & 0.34 & 0.17 \\
\hline Summed Resting Score (SRS) & $\begin{array}{l}2.88 \pm 5.6 \\
6\end{array}$ & $\begin{array}{l}2.18 \pm 5.1 \\
9\end{array}$ & $\begin{array}{l}1.44 \pm 3.6 \\
3\end{array}$ & 0.97 & 0.2 & 0.7 & 0.18 \\
\hline Summed Difference Score (SDS) & $\begin{array}{l}0.87 \pm 1.7 \\
9\end{array}$ & $0.99 \pm 1.9$ & $\begin{array}{l}0.78 \pm 1.8 \\
3\end{array}$ & 1.0 & 1.0 & 1.0 & 0.64 \\
\hline
\end{tabular}


Figure 1. Linkage Disequilibrium

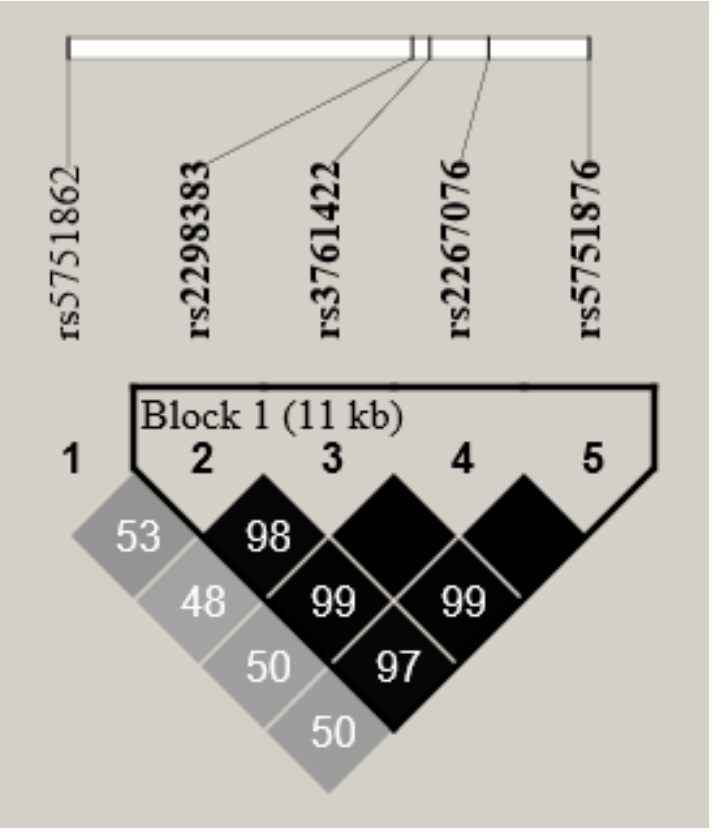




\section{Legends:}

Figure 1.

Shades of grey and numbers indicate extent (D') of linkage disequilibrium (LD) with haploblock assignment. (black/empty box: D'=1). 